\title{
Research on Wide-band Vibration energy Harvester Based on Piezoelectric Effect
}

\author{
Jiansheng Cui ${ }^{1}$, Hongcheng Yin ${ }^{1}$, Sikun Heng ${ }^{1}$, Yunfeng Qiu ${ }^{1}$, Nengpeng Wang ${ }^{1}$ and Yuansheng Chen ${ }^{2}$ \\ ${ }^{1}$ State Grid Jiangsu Power Company Lianyungang Power Company, Nanjing 222002, China \\ ${ }^{2}$ School of Energy and Power Engineering, Nanjing University of Science and Technology, Nanjing 210094, China
}

\begin{abstract}
Vibration energy is a kind of energy form which is ubiquitous in living environment. By using the piezoelectric principle of piezoelectric ceramics, it can design a kind of vibration energy recovery device which can effectively transform the vibration energy in the environment. Because the fluctuation frequency of vibration generated by different vibration sources in the external environment is different, this paper starts from the broadband research, and uses the arrangement of the transducer array to widen the frequency band for the piezoelectric energy acquisition device. In this paper, the finite element analysis software ANSYS is used to model the piezoelectric vibrator structure of different configurations. The static analysis and modal analysis are carried out in this order. The simulation results are obtained and analyzed. And then use the harmonic response analysis, the results of the verification. Finally, based on the standard energy acquisition circuit, this paper studies the interface circuit which is suitable for the vibration model and improves the output power so that the vibration energy recovery device can work normally after the band is widened and output stable power for power supply. This study provides the basis for realizing the power supply of intelligent sensor.
\end{abstract}

Keywords-vibration energy recovery; wide-band vibration; piezoelectric vibration; intelligent sensor

\section{INTRODUCTION}

Vibration energy is a kind of energy widely existing in nature. People pay more and more attention to how to use vibration energy to supply power for electronic devices and realize energy self-sufficiency. There are three mechanisms that can be used for vibrational energy collection: the use of electrostatic devices, electromagnetic fields, and the use of piezoelectric materials. Compared with electrostatic devices and electromagnetic fields, the use of piezoelectric materials has the advantages of high energy output per unit time, no influence of electromagnetic effects, no need for additional power systems, and is currently the main research direction of vibration energy collection research.

Piezoelectric energy harvester is one of the core devices for energy conversion. Its performance directly determines the output energy size and energy conversion efficiency. Piezoelectric energy collectors cause the piezoelectric oscillator structure to deform due to vibrations in the environment. According to the characteristic of the piezoelectric ceramic's unique strain and electric displacement, an $\mathrm{AC}$ voltage with similar vibration frequency is generated on the piezoelectric sheet, and after the AD-DC power conversion, the required electrical energy is provided for the electronic equipment. Therefore, compared with the other two methods, the external requirements for power generation using piezoelectric materials are lower, and the application prospects are even broader.

The piezoelectric vibrator structure has been rapidly developed and many different types of structures have been developed. Among them, the rectangular single cantilever structure is the most commonly used structural form in the current cantilever structure due to its simple structure and easy fabrication. It is also the main research direction of this paper.

At present, more and more studies on broadband, multi-directional, and high-efficiency vibration energy harvesting devices have been conducted at home and abroad. A cantilever beam piezoelectric energy harvester developed by the Australian National University Canberra uses a model with a mass at the end of a cantilever beam for use in microscale electronic devices. It can work under external load with a frequency of about $115 \mathrm{~Hz}$, and the output power can reach $350 \mu \mathrm{W}$ or more, which can make the continuous operation of miniature electronic components continuously.

Taiyuan University of Technology designed a first-order and second-order drive low-frequency piezoelectric vibration energy harvester based on micro-electromechanical systems (MEMS).The second-order driving beam of the transmission beam can obtain two ultra-low frequency voltage peaks of $10.98 \mathrm{~Hz}$ and $44.52 \mathrm{~Hz}$ under a certain acceleration condition, so that the operating frequency of the system is reduced even lower, and the effective operating bandwidth below $50 \mathrm{~Hz}$ is wider and more. Suitable for matching with low frequency vibration environment for energy harvesting.

\section{General Model of Cantilever Beam PIEZOELECTRIC VIBRATOR}

Since the piezoelectric ceramic itself is very hard and very brittle, the strain displacement is small. Piezoelectric ceramic itself is generally not directly used as a device, usually combined with the metal elastomer to form a vibrating body, with the advantages of not easy to break, large strain displacement, fast response and so on.

The support mode is one of the decisive factors influencing the energy generation efficiency of the composite piezoelectric vibrator. Different support modes, device operating modes, energy output modes and conversion efficiency vary widely. The cantilever-type piezoelectric oscillator structure support mode is a cantilever support mode. As shown in Fig. 1, this kind of support method can produce 
the greatest deformation, and support the piezoelectric vibrator in a fixed support form to make a back-and-forth motion. The number of periodic motions per unit time is also lower than other methods. Therefore, it is less difficult to collect vibration energy in the external environment.

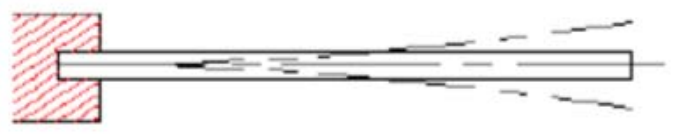

FIGURE I. CANTILEVER SUPPORT MODEL

In this structure, the fixed end portion of the piezoelectric vibrator is usually connected to the support body around the vibrator, and the mass is attached to the free end to form a spring mass system. This kind of vibration combination has a relatively large deformation and is suitable for a low pressure and low frequency environment.

\section{SIMULATION ANALYSIS AND EXPERIMENTAL RESEARCH OF PIEZOELECTRIC VIBRATORS}

\section{A. Finite Element Simulation Analysis}

Since the rectangular cantilever structure is used in this paper, it is more convenient to use a top-down modeling method. Using the voxels already included in ANSYS, such as rectangles, circles, hexahedrons, etc., by using the block command of the diagonal point generation model, the expected diagonal coordinates of the model are directly input, and rectangle models of different sizes are generated, and finally passed. The booleans operation completes the bonding. In the static analysis, we first select a substrate with a length, width, and thickness of $60 \mathrm{~mm}, 20 \mathrm{~mm}$, and $0.5 \mathrm{~mm}$. The fixed end is fixed to a fixed plane. The model is shown in Figure 2.

After the geometric entity of the model is established, after the material is defined and partially adjusted, the network division begins. This article mainly sweeps the body to divide the grid, use the splitter to make the grid unit side length control in $2 \mathrm{~mm}$. The grid density setting interface in the splitter is shown in Fig. 2. Using the grid division method, a hexahedral cell grid is generated for the model, as shown in Fig. 3

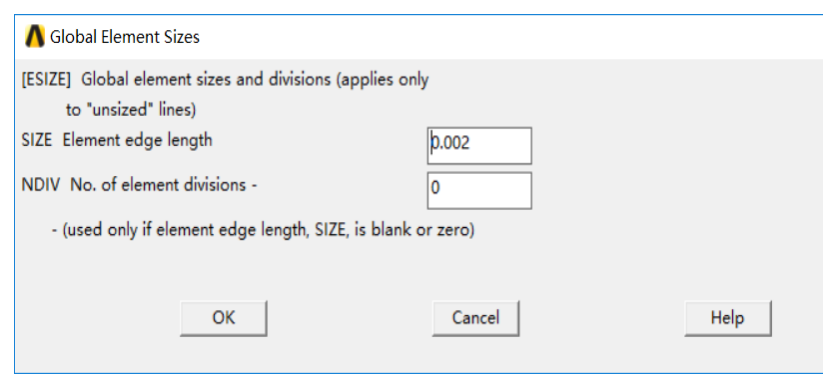

FIGURE II. GRID UNIT SIDE LENGTH CONTROL

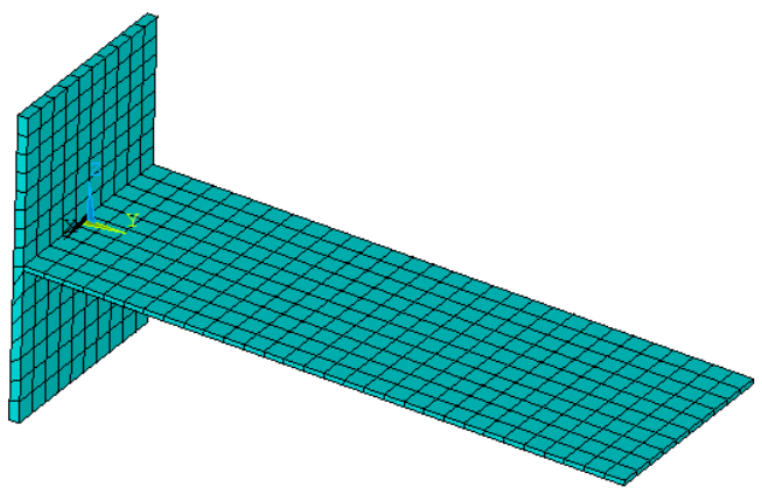

FIGURE III. NETWORK DIVISION

After the network is divided, the fixed end is subjected to displacement constraints. In this paper, the fixed positions of the cantilever beams are each constrained by degrees to keep the end surfaces fixed. Then follow the corresponding analysis and solution to the model to get the analysis results.

\section{B. Static Analysis}

The ANSYS finite element software was used to study the piezoelectric ceramics and substrate on the cantilever beam. The static analysis was first performed and the $5 \mathrm{~mm}$ displacement constraint along the $\mathrm{Y}$ axis was applied to the free end of the cantilever substrate of the piezoelectric ceramic plate. Determine the stress distribution of the cantilever beam, according to the formula of the piezoelectric equation, determine the optimal placement of the ceramic sheet. As can be seen from Figure 4, after applying a displacement constraint of $5 \mathrm{~mm}$ at the free end, the maximum stress at the fixed end portion is $314 \mathrm{MPa}$, and the minimum stress at the free end is only $456 \mathrm{~Pa}$. According to the piezoelectric equation, the maximum electric strength can be obtained at the place where the stress is the highest. Therefore, the piezoelectric ceramic should be placed next to the fixed plate and placed at the root position of the beam.
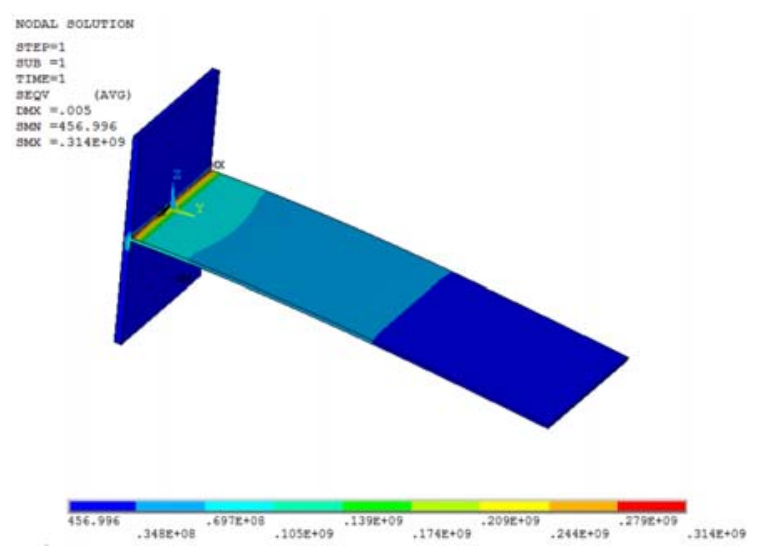

FIGURE IV. CANTILEVER BEAM STRESS DISTRIBUTION

C. Influence of the length of ceramic piece on the natural frequency and stress of the structure

In order to study the influence of the length of the ceramic piece on the natural frequency and the generated stress of the 
piezoelectric vibrator structure, a bronze substrate with a length, width and thickness of $100 \mathrm{~mm}, 20 \mathrm{~mm}$, and $1 \mathrm{~mm}$ was selected. In combination with piezoelectric ceramics of different lengths, the width and thickness of PZT-5H piezoelectric ceramics are $20 \mathrm{~mm}$ and $0.5 \mathrm{~mm}$, respectively. Seven piezoelectric ceramics with $30 \mathrm{~mm}, 40 \mathrm{~mm}, 50 \mathrm{~mm}, 60$ $\mathrm{mm}, 70 \mathrm{~mm}, 80 \mathrm{~mm}, 90 \mathrm{~mm}$, and $100 \mathrm{~mm}$ lengths of piezoelectric ceramics were selected for simulation. The experimental process is shown in Figure 5. A fixed constraint is applied to the fixed end of the composite vibrating body, and then modal analysis is performed. The first-order natural frequency of each piezoelectric vibrator is measured, and the stress generated by the vibration at the natural frequency is measured.

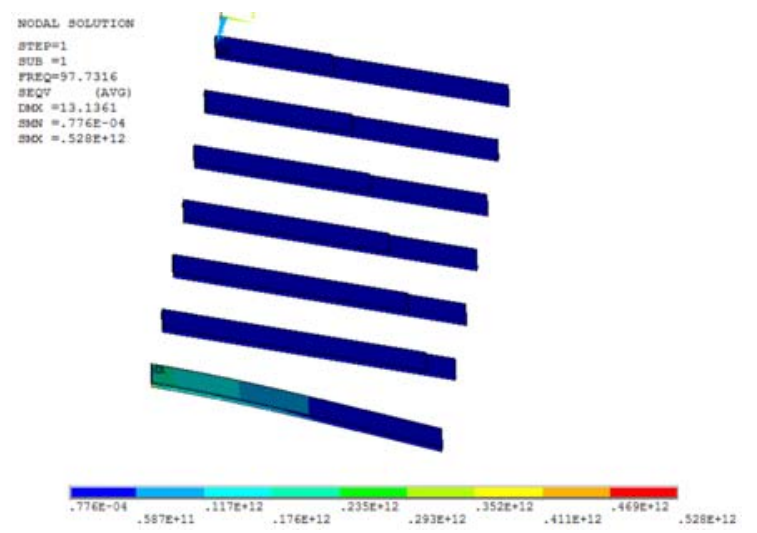

FIGURE V. STRESS MAP OF A 100 MM LONG CERAMIC PIECE

Figure 6 shows that as the length of the ceramic piece increases, the natural frequency first increases and then decreases, but the change in the overall natural frequency is not obvious, and the effect of the length of the ceramic piece on the natural frequency of the piezoelectric vibrator is not Big. From Fig. 3 and Fig. 7, it can be seen that the maximum stress is generated at the fixed end of the piezoelectric vibrator, and the maximum stress increases first and then decreases with the change in the length of the ceramic piece. Therefore, if the length of the piezoelectric ceramic is too long or too short, it is not conducive to the collection of energy. The optimum size is $1 / 2$ to $2 / 3$ of the length of the substrate.

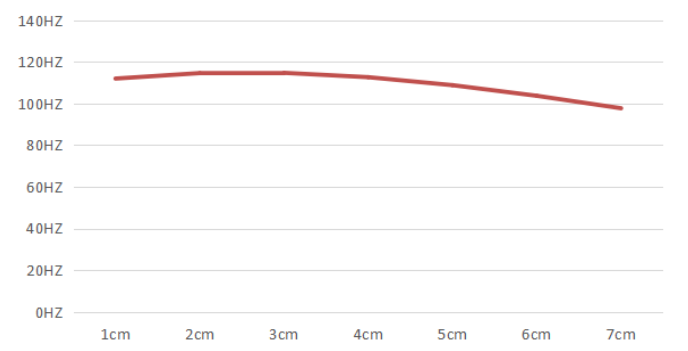

FIGURE VI. CHANGE OF NATURAL FREQUENCY WITH THE LENGTH OF CERAMIC

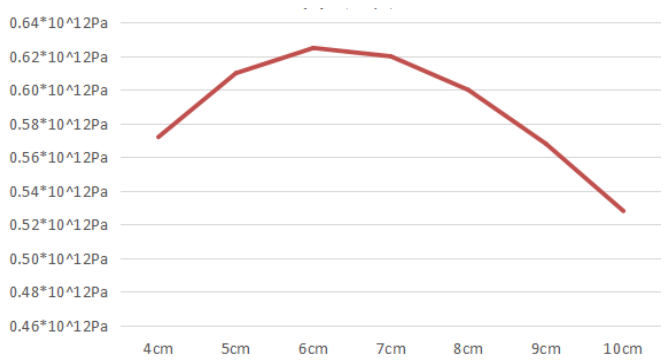

FIGURE VII. THE RELATIONSHIP BETWEEN THE MAXIMUM STRESS AND THE LENGTH OF THE CERAMIC PIECE

\section{Substrate Size and Effect of Increasing Mass on Natural Frequency}

From the above analysis, it can be known that the influence of the ceramic piece length on the natural frequency of the structure is not so great. Under the above-mentioned selected substrate size, the first-order natural frequency is too high and is not suitable for practical applications. In practical applications, the width and thickness of the ceramic sheet generally have certain specifications and it is difficult to make adjustments in parameters. Therefore, the influence of the size of the substrate and the size of the mass on the natural frequency is studied in this paper.

A ceramic substrate with a thickness of $1 \mathrm{~mm}$ and a width of $20 \mathrm{~mm}$ and a width and thickness of $20 \mathrm{~mm}$ and $0.5 \mathrm{~mm}$ was selected. The substrate was suspended $40 \mathrm{~mm}$ along the length of the piezoelectric ceramic and combined with the ceramic sheet to form a composite vibrating body. Five groups of substrates with lengths of $80 \mathrm{~mm}, 90 \mathrm{~mm}, 100 \mathrm{~mm}$, $110 \mathrm{~mm}$, and $120 \mathrm{~mm}$ were selected for simulation. The result is shown in Figure 8.

As can be seen from the figure, when the width and thickness of the substrate and the piezoelectric sheet remain constant, the natural frequency decreases as the length of the substrate increases.

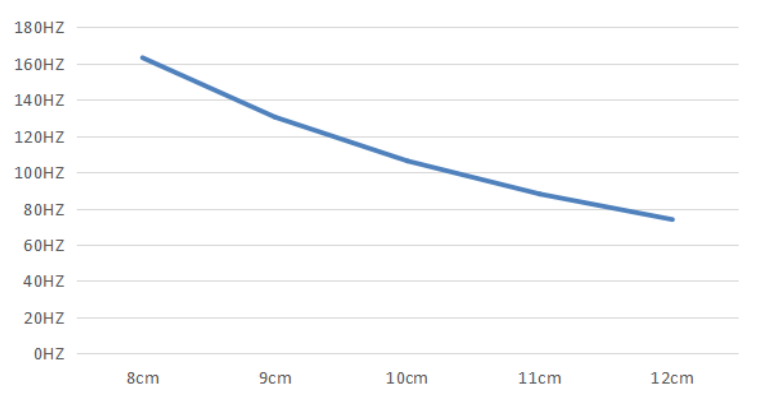

FIGURE VIII. RELATIONSHIP BETWEEN NATURAL FREQUENCY AND SUBSTRATE LENGTH

The selected length and thickness of the substrate is 80 $\mathrm{mm}, 1 \mathrm{~mm}$, and the length and thickness of the ceramic sheet are $40 \mathrm{~mm}$ and $0.5 \mathrm{~mm}$, respectively. Four groups of vibrating bodies with substrate widths of $10 \mathrm{~mm}, 15 \mathrm{~mm}, 20 \mathrm{~mm}$, and $25 \mathrm{~mm}$ were selected for simulation. The result is shown in Figure 9.

As can be seen from the figure, the natural frequency of the piezoelectric vibrator does not substantially change with 
an increase in the width of the substrate. Therefore, it can be considered that the natural frequency of the piezoelectric vibrator is independent of the width of the substrate and the piezoelectric sheet.

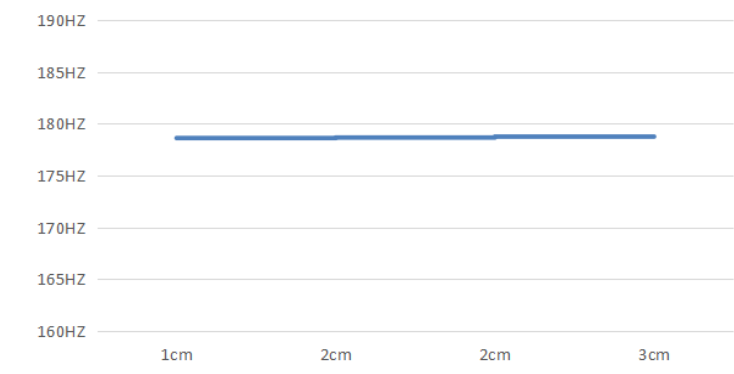

FIGURE IX. RELATIONSHIP BETWEEN NATURAL FREQUENCY AND SUBSTRATE WIDTH

Ceramic plates of $40 \mathrm{~mm}, 20 \mathrm{~mm}$, and $0.5 \mathrm{~mm}$ in length, width, and thickness were selected, with a substrate length of $80 \mathrm{~mm}$ and a width of $20 \mathrm{~mm}$. Five groups of substrates with thicknesses of $1 \mathrm{~mm}, 1.5 \mathrm{~mm}, 2 \mathrm{~mm}, 2.5 \mathrm{~mm}$, and $3 \mathrm{~mm}$ were selected for simulation. The result is shown in Figure 10.

As can be seen from the figure, in the case where the length and the width of the substrate and the piezoelectric sheet remain unchanged, the natural frequency increases as the thickness of the substrate increases, but the changing tendency does not change linearly. In addition, the thicker the substrate thickness, the smaller the resonance amplitude of the piezoelectric composite vibrator, which is not conducive to energy acquisition. Therefore, we generally keep the thickness of the material constant and change other parameters to adjust the natural frequency.

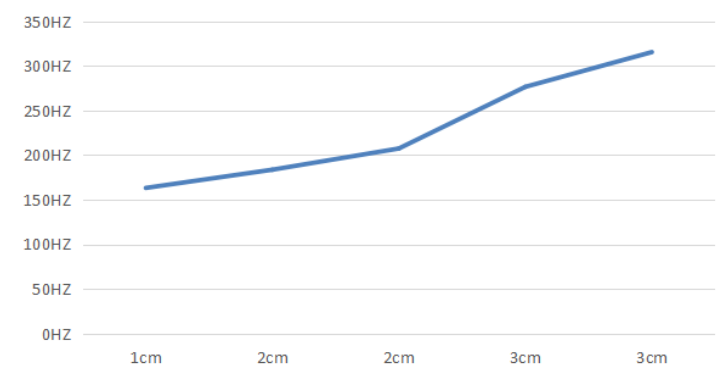

FIGURE X. RELATIONSHIP BETWEEN NATURAL FREQUENCY AND SUBSTRATE THICKNESS

We know that the mass distribution of the model has a great influence on the natural frequency. Therefore, we place a mass at the free end of the vibrator. By changing the mass of the mass, we can determine the natural frequency change trend of the model under different mass distributions. First, the length, width and height of the ceramic piece were selected as $40 \mathrm{~mm}, 20 \mathrm{~mm}$ and $0.5 \mathrm{~mm}$ ceramic pieces. The length, width and height of the ceramic piece were selected as $80 \mathrm{~mm}, 20 \mathrm{~mm}$ and $1 \mathrm{~mm}$. Five groups of different masses were selected for experiments. The masses were $5 \mathrm{~g}, 10 \mathrm{~g}, 15 \mathrm{~g}$, $20 \mathrm{~g}$, and $25 \mathrm{~g}$, respectively, to investigate the effect of mass distribution on the natural frequencies of the piezoelectric vibrators. The result is shown in Figure 11.
From the figure, we can see that by changing the size of the mass, as the mass increases, the natural frequency of the vibrator decreases rapidly. It can be seen from the curve that the relationship between them is not linear. Therefore, adjusting the mass does not control frequency changes well. However, the increase of the mass can effectively reduce the natural frequency of the vibrator to achieve the change of the frequency band, which is of great significance for practical use.

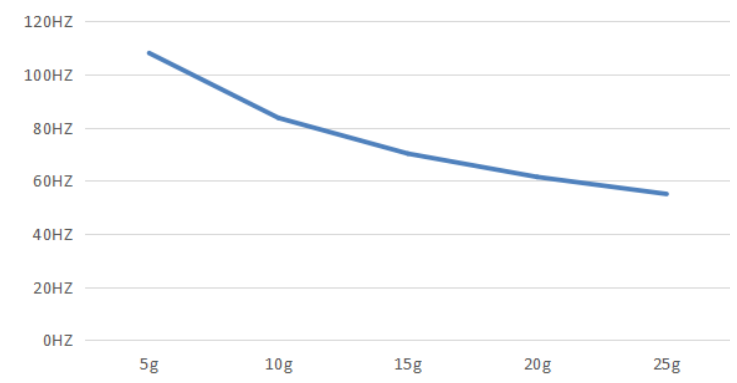

FIGURE XI. EFFECT OF MASS ON NATURAL FREQUENCY

In order to intuitively add the changes in the natural frequency before and after the mass, we select the above-mentioned models with more regular natural frequency variation curves to better reflect the experimental results. The change in length of the influence of the change in length on the natural frequency is relatively stable. We chose this group of controlled experiments and based on the first set of experiments, we added a length, width and height of $20 \mathrm{~mm}$ to the free end of the cantilever beam for each experimental sample. $6 \mathrm{~mm}, 1 \mathrm{~mm}$ rectangular mass, and the mass density is defined as $25000 \mathrm{~kg} / \mathrm{m} 2$, the elastic modulus takes a very large value, so that the mass near the rigid body model, you can get a $30 \mathrm{~g}$ mass. Then follow the simulation steps of the above-mentioned group control experiment to obtain the natural frequency under the mass with different lengths of the substrate, as shown in fig. 12 .

From the curves, we can see that whether or not there are mass blocks has a great influence on the natural frequency of the piezoelectric vibrator, and the change in the length and thickness of the substrate is far from increasing the apparent mass. In practice, the length and thickness of the substrate will be affected. Therefore, in practical applications, we add a certain mass of mass at the free end of the cantilever beam, which can effectively reduce the natural frequency and reduce the frequency band to the required frequency range. At the same time, in other studies, it has been repeatedly mentioned that increasing the mass can increase the energy output. The application of mass blocks will provide many benefits for broadband design. 


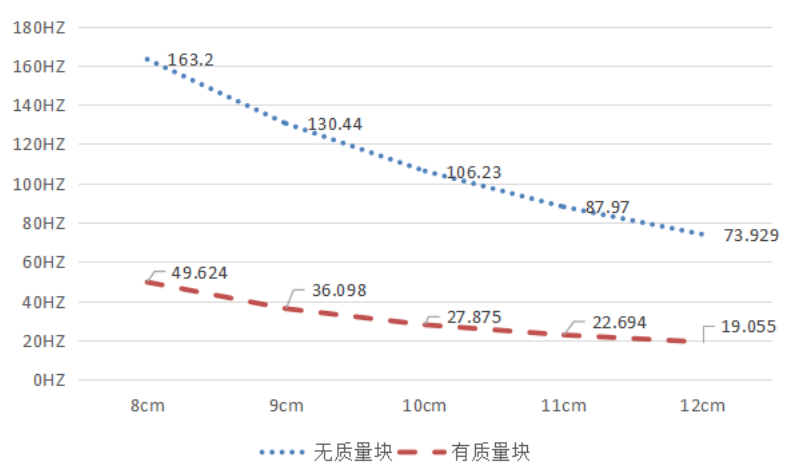

FIGURE XII. ADDITIONAL QUALITY COMPARISON CHART

\section{E. Research and Finite Element Analysis of Broadband Piezoelectric Energy Harvesters}

From the above analysis, a plurality of factors affecting the natural frequency of the cantilever-type piezoelectric vibrator can be obtained by the piezoelectric vibrator structure. From this, we can experimentally study the wide-band piezoelectric energy collector based on known experimental results.

In the experiment, the array is used to widen the frequency band of the collector to solve the problem that the collector frequency band is too narrow in the practical application, resulting in a lower energy output efficiency and less output.

1) Modal analysis: Because plexiglass has the advantages of high mechanical strength, light weight, and easy processing, this material was chosen as the matrix of the cantilever beam array. The simulated broadband model in the experiment is shown in Figure 13. Five groups of cantilever composite vibrators were selected. The lengths of the substrates were 80 $\mathrm{mm}, 100 \mathrm{~mm}, 120 \mathrm{~mm}, 140 \mathrm{~mm}$, and $160 \mathrm{~mm}$, respectively. The length of the ceramic plate was half the length of the substrate. The width and thickness of the ceramic sheet are 20 $\mathrm{mm}$ and $0.5 \mathrm{~mm}$. The material is PZT-5H. The width and thickness of the substrate are $20 \mathrm{~mm}$ and $1 \mathrm{~mm}$. The material is a bronze sheet. The mass of the mass is $15 \mathrm{~g}$, and the substrate is $100 \mathrm{~mm}, 20 \mathrm{~mm}$, and $4 \mathrm{~mm}$ plexiglass.

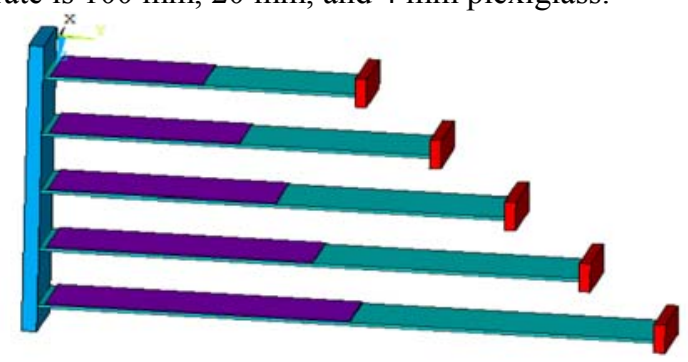

FIGURE XIII. BROADBAND PIEZOELECTRIC ENERGY HARVESTER

After completing the model and dividing the mesh, a fixed constraint is applied on the vertical surface of the substrate and then the modal analysis is performed. The results are shown in Figure 14. The first-order natural frequencies of the cantilever array are $19.055 \mathrm{~Hz}, 22.694 \mathrm{~Hz}, 27.875 \mathrm{~Hz}, 36.098$ $\mathrm{Hz}$, and $49.624 \mathrm{~Hz}$. The device can effectively collect energy when the device is shaken at a frequency of $20-50 \mathrm{~Hz}$, so that the piezoelectric energy collection device can reach a suitable frequency bandwidth, which is favorable for improving the ability of the power generation device to adapt to the environment and improve the output.

In order to eliminate other factors brought about by the combined model, a comparative test of the assembled body and the individual cantilever beam was performed. From the figure, it can be seen that the combined frequency of the cantilever beam and the single cantilever beam structure is basically the same as the length of the substrate. The cantilever beam array model can be used to broaden the frequency band, but the natural frequency of each cantilever beam is reduced to a certain extent. After the additional matrix combination, the shape of the model and the mass distribution on the material are changed. Therefore, the natural frequency has decreased. In the actual design, it is necessary to pay attention to this point.

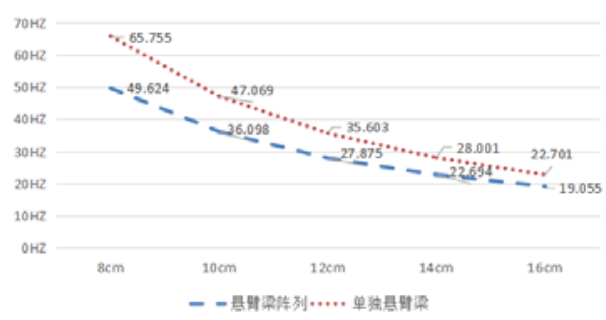

FIGURE XIV. COMPARISON OF NATURAL FREQUENCY OF CANTILEVER BEAM ARRAY AND SINGLE CANTILEVER BEAM

2) Harmonic response analysis: Harmonic response analysis is mainly used to analyze the continuous periodic load in the system and determine the model output changes over time when the model is subjected to harmonic load. At present, there are mainly the following methods to analyze: Full (complete method), Reduced (mode reduction method), and Mode superposition (mode superposition method). In this paper, the complete method is used to analyze the model.

For the above wide-band piezoelectric energy harvester, a periodic load of $20 \mathrm{~N}$ in the Z-axis direction was applied to the piezoelectric vibrator, and the free end of the piezoelectric vibrator was used as an observation point to perform harmonic response analysis. The results are shown in fig. 15 . Verify that the above device has the possibility of broadband energy harvesting.

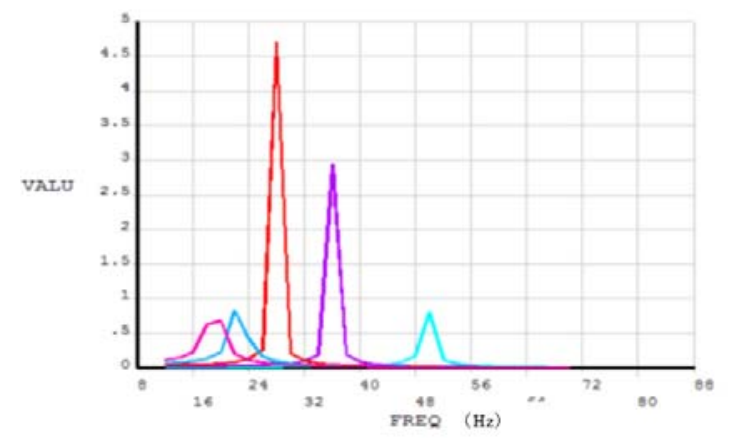

FIGURE XV. 15 HARMONIC RESPONSE ANALYSIS RESULTS 
From the analysis results, it can be seen that when the frequency reaches $19 \mathrm{~Hz}, 23 \mathrm{~Hz}, 28 \mathrm{~Hz}, 36 \mathrm{~Hz}$, and $50 \mathrm{~Hz}$, the vibrator can reach the maximum displacement value and produce the maximum strain. Therefore, in the natural frequency of each order, the vibrator generates resonance and is in the best working condition. And in $20-50 \mathrm{~Hz}$, there are multiple overlapping output curves, which proves that the device can collect vibration energy from different vibration sources in a band.

From the above analysis, it can be seen that the cantilever array structure is of great significance to broadening the frequency band, the cantilever array has a simple structure, is easy to perform data analysis, has a wide frequency band adjustment range, and has many adjustment methods. It is suitable to make adjustments in different environments to meet different energy collection requirements to improve energy harvesting capabilities.

\section{RESEARCH ON INTERFACE CiRCUIT OF BROADBAND Piezoelectric EnERgy HaRVESTING DeVICE}

\section{A. Interface Circuit Schematic}

At present, the interface circuits used for the wide-band type piezoelectric energy harvesting device mainly include the following four methods to combine the output power of the piezoelectric slices. Respectively, after the first parallel rectifier circuit, after the first series rectifier circuit, the first rectification after the parallel circuit, the first rectification after the series circuit. In the following article, four types of circuits will be simulated separately, and the power output waveforms will be obtained and compared to draw conclusions.

Since the piezoelectric sheet is resonant at the natural frequency, the oscillator structure is displaced according to the sine law. Therefore, in the simulation, the output waveform of the oscillator structure is a sine wave type. We use the first-order natural frequencies of the cantilever array as 19.055 $\mathrm{Hz}, 22.694 \mathrm{~Hz}, 27.875 \mathrm{~Hz}, 36.098 \mathrm{~Hz}$, and $49.624 \mathrm{~Hz}$ as the output frequencies of the five piezoelectric slices. The clamp capacitance values for piezoelectric ceramics are assumed to be $40 \mathrm{nF}, 50 \mathrm{nF}, 60 \mathrm{nF}, 70 \mathrm{nF}, 80 \mathrm{nF}$, and the output currents are assumed to be $100 \mu \mathrm{A}, 120 \mu \mathrm{A}, 140 \mu \mathrm{A}$, and $160 \mu \mathrm{A}$. The filter capacitor is $5 \mu \mathrm{F}$ and the load resistance is $100 \mathrm{k} \Omega$. The above component parameters are selected for comparison experiments. After proper debugging, the load waveforms are stable within $3 \mathrm{~s}$. Therefore, we select the running time as 3 seconds to view the results. See Appendix A for the schematic of the four circuits.

\section{B. Simulation Results and Analysis}

See Appendix B for simulation results. The two groups are first analyzed after the bridge-type full-control rectification followed by serial and parallel circuits, and then compared with the other two groups after the rectification circuit. The results show that the circuit of rectification, restringing and paralleling is the first, and the output power is obviously larger than that of directly connecting the oscillators in series or in parallel. The output waveform is stable and the fluctuation is small, which effectively reduces the internal energy consumption of the circuit and improves the output power.

From the above analysis, it can be seen that the circuit that performs rectification work first has better performance, higher output power, and more stable output. So this text carries on further analysis to these two groups of circuits. From the figure, it can be seen that, in the circuit in which the vibrator is connected in parallel, the power output has a stable value of $630 \mu \mathrm{W}$, and the output waveform is stable after $1 \mathrm{~s}$, and the waveform fluctuation is small. In the series circuit of the vibrator, the power output has a stable value of $500 \mu \mathrm{W}$. The output waveform stabilizes after $2 \mathrm{~s}$, and the waveform has small fluctuations. Through analysis, it can be seen that the parallel output circuit of the vibrator has a large output power, fast response, less fluctuation, and stable output. In practical applications, this kind of circuit has higher output efficiency.

\section{CONCLUSION}

In this paper, we first study the basic theory of piezoelectric properties, basic concepts such as vibration structure and interface circuit. Then we introduce the basic theory of the cantilever beam piezoelectric vibrator, and cite the three main structural design methods for widening the current band, in the cantilever beam. Based on the related basic theories of piezoelectric vibrators, four broadband interface circuits are introduced to provide a certain interface circuit design theory.

(1) Using ANSYS to perform finite element analysis of piezoelectric vibrators. According to the static analysis results, the maximum electric strength can be obtained at the place where the stress is the highest. Therefore, the piezoelectric ceramic should be placed next to the fixing plate and placed at the root position of the beam. According to the results of modal analysis, the effect of the different structural dimensions of the material on the natural frequency was obtained. Therefore, it can be seen that the cantilever array structure can effectively broaden the frequency band.

(2) Experimental study of a wide-band piezoelectric energy harvester. In the experiment, using the array method, the frequency band broadens. The designed device has multiple overlapping output curves within 20-50 Hz. The harmonic response analysis can be used. At each order natural frequency, the resonator generates resonance and produces the maximum Displacement. The output curves have multiple overlaps, which proves that the device can collect vibrational energy from different vibration sources in a certain frequency band, proving that the device can collect vibrational energy from different vibration sources in a certain frequency band, effectively widen the frequency band, and increase output capacity. .

(3) Based on the standard energy harvesting circuit, this paper studies four kinds of interface circuits suitable for the vibration model. The results show that the circuits that are rectified and then re-stringed and paralleled are better than the first and parallel rectified circuits. In the two circuits that are first rectified, then re-stringed and paralleled, the circuit in the form of a parallel-connected vibrator has a larger output 
power than a series connection, and has a faster response and is more suitable for practical applications.

\section{ACKNOWLEDGMENT}

This study was supported by National Natural Science Foundation of China (No. 51607091, 51405235); Fundamental Research Funds for the Central Universities (No.30916011334); State Key Laboratory of Smart Grid Protection and Control (No.SGNR0000GZJS1705881).

\section{REFERENCES}

[1] Kim H. W. , Batra A. , Priya S. , et al. Energy harvesting using a piezoelectric cymbal transducer in dynamic environment. Japan Journal of Applied Physics , 2004 , 43 (6): 178 - 183.

[2] Lefeuvre E. , Sebald G. , Guyomar D. , et al. Materials , structures and power interfaces for efficient piezoelectric energy harvesting. Journal of Electroceram , 2007, 22(1-3): 171-179

[3] Lefeuvre E. , Badel A. , Richard C. , et al. A comparison between several vibration-powered piezoelectric generators for standalone systems. Sensors and Actuators A , 2006 , 126(2): 405-416.

[4] Han J., Von Jouanne A., Le T, et al. Novel power conditioning circuits for piezoelectric micro power generators. Proc. 19 th Ann. IEEE App lied Power Electronics Conf. And Exposition Conf , 2004 , 1541 1546.

[5] Lesieutre G. A., Ottman G. K., Hofmann H. F. Damping as a result of piezoelectric energy harvesting. Jounal of Sound and Vibration , 2004 , 269 (3): 991 - 1001.

[6] Lefeuvre E. , Audigier D. , Richard C. , et al. Buck-boost converter for sensorless power optimization of piezoelectric energy harvester. IEEE Transaction on Power Electronics , 2007 , 22(5): 2018-2025.

[7] Shenck N. S. and Paradiso J. A. Energy scavenging with shoe-mounted piezoelectrics IEEE Micro , 2001 , 21:30-42

[8] Ammar Y., Buhrig A. , Marzencki M. , et al. Wireless sensor network node with asynchronous architecture and vibration harvesting micro power generator. Proc. 2005 Joint Conf. on SmartObjects and Ambient ibeuntelligence: Innovative Context2Aware Services: Usages and Technologies(Grenoble) , 2005, 287 - 292.

[9] Lefeuvre E. , Badel A. , Richard C. , et al. Piezoelectric energy harvesting device optimization by synchronous electric charge extraction. Journal of Intelligent Material Systems and Structures , 2005 16(10): 865-876

[10] Guyomar D. , Badel A. , Lefeuvre E. , et al. Toward energy harvesting using active materials and conversion improvement by nonlinear processing IEEE Trans. Ultrason. Ferroelectr. Freq. Control , 2005, 52:584-94

[11] Badel A. , Guyomar D. , Lefeuvre E. , et al. Efficiency enhancement of a piezoelectric energy harvesting device in pulsed operation by synchronous charge inversion. Journal of Intelligent Material Systems and Structures , 2005 , 16: 889 - 901 .

[12] Lefeuvre E., Badel A., Benayad A., et al. A comparison between several approaches of piezoelectric energy harvesting. Physique Coll ,2005 , 128: $177-186$

[13] Lallart M, Garbuio L, Petit L, et all. Double Synchronized Switch Harvesting (DSSH): A new energy harvesting scheme for efficient energy extraction. Ultrasonics, Ferroelectrics and Frequency Control, IEEE Transactions on, 2008, 55(10):2119 2130.

[14] Shen H, Qiu J, Ji H, et all. Enhanced synchronized switch harvesting: A new energy harvesting scheme for efficient energy extraction. Smart materials and structures, 2010,19(11): 115017. 